\title{
Analyzing the Mechanisms Behind Macrolide Antibiotic-Induced Liver Injury Using Quantitative Systems Toxicology Modeling
}

\author{
Jeffrey L. Woodhead ' Kyunghee Yang' - David Oldach ${ }^{2}$ - Chris MacLauchlin ${ }^{2}$. \\ Prabhavathi Fernandes ${ }^{2} \cdot$ Paul B. Watkins ${ }^{3} \cdot$ Scott Q. Siler ${ }^{\prime} \cdot$ Brett A. Howell $^{\prime}$
}

Received: 24 September 2018 / Accepted: 27 January 2019 / Published online: 7 February 2019

(C) The Author(s) 2019

\begin{abstract}
Purpose Macrolide antibiotics are commonly prescribed treatments for drug-resistant bacterial infections; however, many macrolides have been shown to cause liver enzyme elevations and one macrolide, telithromycin, has been pulled from the market by its provider due to liver toxicity. This work seeks to assess the mechanisms responsible for the toxicity of macrolide antibiotics.
\end{abstract}

Methods Five macrolides were assessed in in vitro systems designed to test for bile acid transporter inhibition, mitochondrial dysfunction, and oxidative stress. The macrolides were then represented in DILIsym, a quantitative systems pharmacology (QST) model of drug-induced liver injury, placing the in vitro results in context with each compound's predicted liver exposure and known biochemistry.

Results DILIsym results suggest that solithromycin and clarithromycin toxicity is primarily due to inhibition of the mitochondrial electron transport chain (ETG) while erythromycin toxicity is primarily due to bile acid transporter inhibition. Telithromycin and azithromycin toxicity was not predicted by DILIsym and may be caused by mechanisms not currently incorporated into DILIsym or by unknown metabolite effects.

Electronic supplementary material The online version of this article (https://doi.org/l 0. I007/s I | 095-0 19-2582-y) contains supplementary material, which is available to authorized users.

Jeffrey L. Woodhead

jwoodhead@dilisym.com

DILlsym Services, Inc., a Simulations Plus Company, 6 Davis Drive, PO Box 12317, Research Triangle Park, North Carolina 27709, USA

2 Cempra, Inc., Chapel Hill, North Carolina, USA

3 UNC Eshelman School of Pharmacy, The University of North Carolina at Chapel Hill, Chapel Hill, North Carolina, USA
Conclusions The mechanisms responsible for toxicity can be significantly different within a class of drugs, despite the structural similarity among the drugs. QST modeling can provide valuable insight into the nature of these mechanistic differences.

KEY WORDS antibiotics . BSEP inhibition - liver injury . mitochondria · quantitative systems toxicology

\section{INTRODUCTION}

The macrolide class of antibiotics are frequently prescribed antibiotics for otherwise drug-resistant bacterial infections $(1,2)$. Several macrolide antibiotics are currently available for clinical use. Erythromycin is the first generation macrolide; it was isolated from the bacteria Saccharopolyspora erythraea in the 1950s. Clarithromycin and azithromycin are the second generation macrolides; they are semi-synthetic derivatives of erythromycin. Erythromycin, clarithromycin, and azithromycin have been reported to cause mild, asymptomatic elevations in serum alanine aminotransferase (ALT) in 1-2\% of the population (3), and are associated with very rare cases of clinically important liver injury (4). Widespread resistance to existing macrolides necessitated development of the next generation of new antibiotics. Telithromycin is a macrolide where the cladinose sugar found in the older macrolides is replaced with a keto group in addition to other changes, thus called a ketolide. Telithromycin showed activity against macrolideresistant strains and was approved by regulatory agencies for marketing in the early 2000s. However, rare cases of serious liver injury including acute liver failure occurred in patients treated with telithromycin (5), which led to a boxed warning about serious liver toxicity and restriction of indication. As a result, telithromycin was voluntarily pulled from the U.S. market by its provider (6,7). After telithromycin, no other macrolide antibiotics have been approved for clinical use in the U.S. 
Solithromycin, a novel macrolide antibiotic and the first fluoroketolide, has been developed to treat moderate to moderately-severe community-acquired bacterial pneumonia $(\mathrm{CABP})$ and otherwise drug-resistant bacterial infections. In multi-center phase III clinical trials with CABP patients, solithromycin showed promise by proving non-inferiority to moxifloxacin $(8,9)$. However, serum ALT elevations occurred with solithromycin at a higher frequency than with other macrolide antibiotics; in phase III clinical trials, 5\% and 9\% of patients developed benign ALT elevations above the 3-fold upper limit of normal (ULN) with the Oral and the IV-toOral protocols, respectively $(8,9)$. Furthermore, solithromycin is structurally similar to telithromycin in also being a ketolide (see Fig. C1 in the supplemental materials), further raising concerns about solithromycin's liver safety profile (10). The FDA has demanded greatly expanded clinical trials of solithromycin to further assess liver safety prior to an approval decision (11).

Quantitative systems toxicology (QST) is a discipline of pharmacology that seeks to understand and ultimately predict the toxic effects of drugs/chemicals by integrating computational and experimental methods (12). DILIsym is a QST model of liver injury which integrates the results from in vitro mechanistic toxicity assays with estimates of in vivo exposure and known biochemistry to understand hepatotoxicity and the biochemical processes behind it (Fig. 1) (13-15). Hepatotoxicity mechanisms represented in DILIsym include oxidative stress, mitochondrial dysfunction, and bile acid transport inhibition, which are mechanistically connected to cell death and ALT elevation through previously described representations of liver biochemistry and physiology (13,14,16-18). Through these mechanisms, DILIsym has successfully predicted hepatotoxic potential of drugs and drug candidates and determined the underlying mechanisms of clinically observed hepatotoxicity signals $(16,17,19)$. In the current study, DILIsym was used to determine the most likely mechanisms behind the ALT elevations observed with five macrolide antibiotics: solithromycin, erythromycin, clarithromycin, telithromycin, and azithromycin. Understanding the mechanisms behind the ALT elevations observed within this drug class could be important in determining whether novel antibiotics might have the same liver safety concerns that scuttled telithromycin.

\section{MATERIALS AND METHODS}

\section{Software Platform}

DILIsym v5A was used to conduct the simulations in this paper. DILIsym is a software package that is available to members of the DILI-sim Initiative; academic and regulatory licensing is also available.

\section{Development of Physiologically-Based Pharmacokinetic (PBPK) Models}

PBPK models for the five macrolide antibiotics were constructed within DILIsym to describe disposition of macrolides in humans. For solithromycin, the PBPK model was based on plasma concentration-time data from clinical trials; literature reports of plasma time courses were used for azithromycin, telithromycin, erythromycin, and clarithromycin. The basic structure of the DILIsym PBPK sub-model has been discussed elsewhere $(13,17,19,20)$; details and results of the PBPK modeling for the five macrolides are provided in Supplement A.

\section{In Vitro Mechanistic Toxicity Assays}

Five macrolides were assessed in in vitro assays for three main hepatotoxicity mechanisms represented within DILIsym: mitochondrial dysfunction, oxidative stress, and bile acid transporter inhibition. To detect potential mitochondrial dysfunction signals, cellular respiration assays were conducted using a Seahorse XFe96 Flux Analyzer in HepG2 cells incubated with various concentrations of macrolides for 1 or $24 \mathrm{~h}$. HepG2 cells were chosen in part because of their metabolic incompetence, since any effect observed in the HepG2 system can be attributed solely to the activity of the parent compound. Induction of oxidative stress was determined by high content screening using a fluorescent probe, dihydroethidium (DHE), in HepG2 cells incubated with various concentrations of macrolides for 1 or $24 \mathrm{~h}$. In these whole cellbased assays, intracellular concentrations of macrolides were determined by LC/MS/MS analysis in parallel HepG2 cultures. Parameter values for macrolide mediated induction of mitochondrial dysfunction and oxidative stress were determined by reproducing the cellular respiration data and the oxidative stress data directly within DILIsym using measured intracellular concentrations. Inhibitory effects of macrolides for bile acid transporters were assessed experimentally using membrane vesicles overexpressing a bile acid efflux transporter (i.e., BSEP, MRP3, or MRP4) and CHO cells overexpressing NTCP or obtained from published literature. Detailed experimental methods are described in Supplement B. Mitochondrial dysfunction and oxidative stress assays were performed by Cyprotex, Inc. (Macclesfield, UK). Transporter inhibition assays were performed by Solvo Biotechnology (Budaors, Hungary). 
Fig. I Quantitative systems toxicology modeling process using DILIsym.

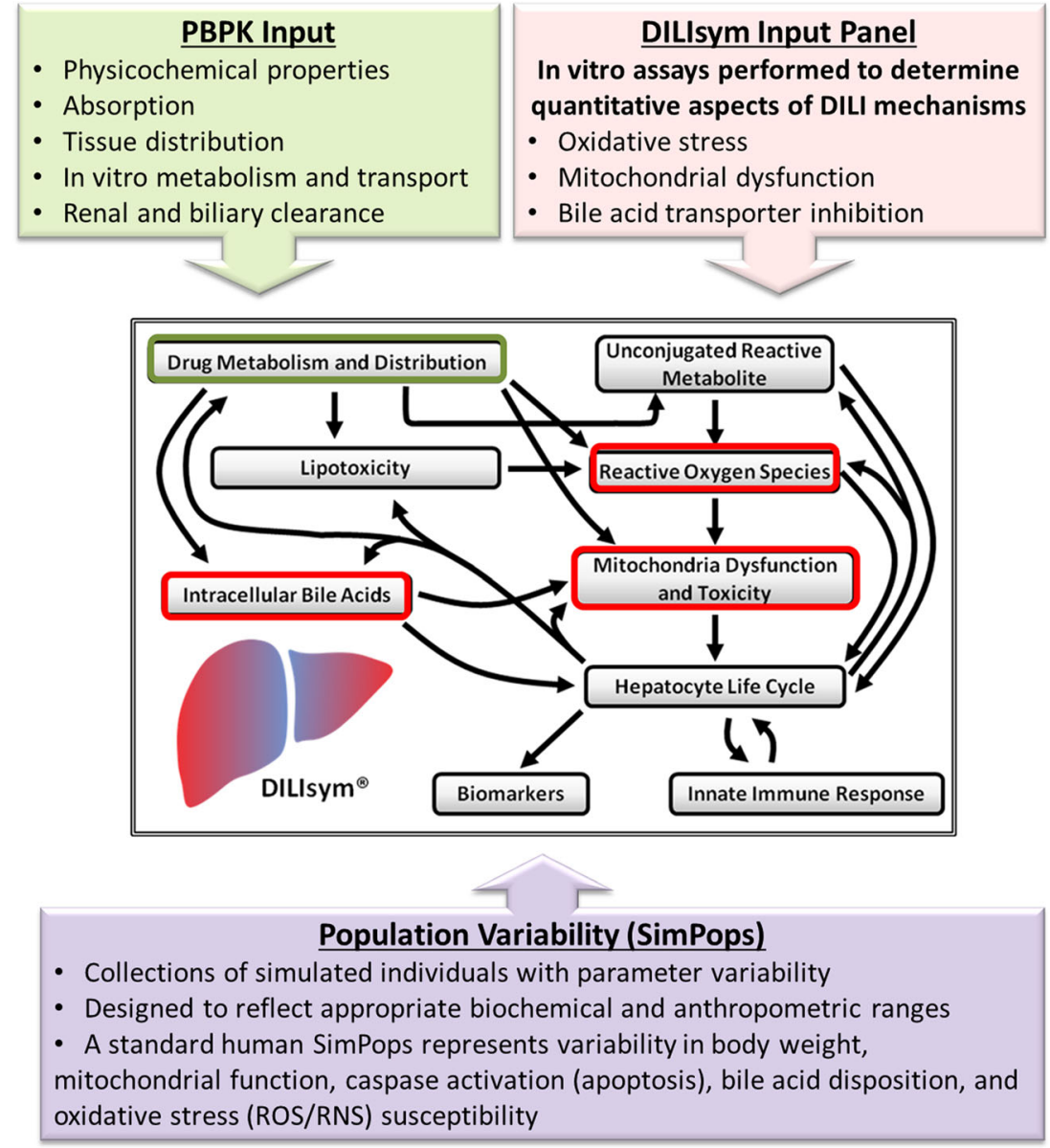

\section{Translation into DILlsym Parameters}

For each of the assays conducted, the results were translated into DILIsym parameters for use in the simulations. The method used for this translation was consistent across compounds. For the bile acid transporter parameters, the $\mathrm{IC}_{50}$ was used directly as the inhibition constant. Mode of inhibition was assumed to be mixed inhibition with $\alpha=5$. While competitive and non-competitive inhibition types may result in low and high extremes of potential bile acid accumulation, respectively, mixed inhibition with $\alpha=5$ leads to a median impact on bile acid accumulation. In addition, mixed inhibitors are more common compared to pure competitive or noncompetitive inhibitors. For mitochondrial dysfunction, the assay results comparing intracellular concentrations and OCR were recapitulated in MITOsym if the OCR decline was non-saturable and in DILIsym if the OGR decline was saturable; the resulting parameters were translated into DILIsym parameters using translation factors involving exemplar compounds, a process which has been reported elsewhere (18). For oxidative stress, the assay results were reproduced using DILIsym by mimicking in vitro conditions; appropriate parameter values for the oxidative stress effects were identified by comparing simulation results with the measured data.

\section{Simulations Conducted}

DILIsym v5A was used to perform simulations on each of the antibiotics at their maximum recommended doses and typical duration of treatment. The protocols used for each drug were as follows:

- Solithromycin Oral protocol: PO $800 \mathrm{mg}$ QD on day 1, and $400 \mathrm{mg}$ QD on days 2-5

- Solithromycin IV-to-Oral protocol: 60-min IV infusion $400 \mathrm{mg}$ QD on days 1-3, PO $800 \mathrm{mg}$ QD on day 4, and PO $400 \mathrm{mg}$ QD on days 5-7

- Erythromycin: PO $500 \mathrm{mg}$ QD, 7 days

- Clarithromycin: PO $500 \mathrm{mg}$ BID, 7 days

- Telithromycin: PO $800 \mathrm{mg}$ QD, 10 days

- Azithromycin: PO $500 \mathrm{mg}$ QD, 7 days 
Table I A List of the Parameters Varied in the v4A_I SimPops Included in DILIsym v5A

Data used to define parameter distributions (if applicable)

\begin{tabular}{ll}
\hline Parameter symbol in DILIsym ${ }^{\circledR}$ & Parameter name in DILIsym ${ }^{\circledR}$ \\
\hline ATP_decr_necrosis_Vmax & ATP decrement necrosis Vmax \\
Body_mass & Body Mass \\
GSH_pre_trans_Vmax & GSH precursor transport Vmax \\
GSHo & GSH basal level \\
HGF_prod_LSEC_Vmax & Maximum LSEC HGF production rate pe \\
HGF_regen_Vmax & HGF mediated regeneration Vmax \\
RNS_ROS_ATP_inhib_Vmax & RNS/ROS ATP inhibition Vmax \\
RNS_ROS_Cl_Vmax & Liver RNS/ROS baseline clearance Vmax \\
Basal_Stdzd_MitoETC_Flux & Basal value of mito ETC flux \\
Resp_Reserve_Scalar & Scaling coefficient representing reserve mitoc \\
function \\
CAS_apop_scale & Caspase-mediated apoptosis scaling constant \\
BA_uptake_Vmax & Bulk bile acid uptake Vmax \\
& \\
BA_baso_Vmax & Bulk bile acid basolateral transport Vmax \\
& \\
BA_canal_Vmax & Bulk bile acid canalicular transport Vmax \\
LCA_uptake_Vmax & \\
& LCA uptake Vmax
\end{tabular}

LCA_baso_Vmax LCA basolateral transport Vmax

LCA_canal_Vmax LCA canalicular transport Vmax

LCAamide_uptake_Vmax LCA-amide uptake Vmax

LCAamide_baso_Vmax LCA-amide basolateral transport Vmax

LCAamide_canal_Vmax LCA-amide canalicular transport Vmax

LCAsulfate_uptake_Vmax LCA-sulfate uptake Vmax

LCAsulfate_baso_Vmax LCA-sulfate basolateral transport Vmax

LCAsulfate_canal_Vmax LCA-sulfate canalicular transport Vmax

Data source for distribution

Assumed standard deviation of $\pm 20 \%$ and parameter range of 2.5 times the S.D. and validated with outcome data

Parameter range from NHANES III (human data)

Parameter range derived from $(2 \mathrm{I})$

Parameter range from $(22,23)$

Assumed standard deviation of $\pm 20 \%$ and parameter range of 2.5 times the S.D. and validated with outcome data

Assumed standard deviation of $\pm 20 \%$ and parameter range of 2.5 times the S.D. and validated with outcome data

Parameter range derived from (24)

Assumed standard deviation of $\pm 20 \%$ and parameter range of 2.5 times the S.D. and validated with outcome data

Parameter range from healthy volunteer data (25)

Parameter range from healthy volunteer data (25)

Parameter range derived from (26)

All transporters were assumed to have the same distribution as human BSEP reported in (27); similar expression ranges are also reported in (28); all uptake Vmax values are covariant

All transporters were assumed to have the same distribution as human BSEP reported in (27); similar expression ranges are also reported in (28); all basolateral Vmax values are covariant

All transporters were assumed to have the same distribution as human BSEP reported in (27); similar expression ranges are also reported in (28); all canalicular Vmax values are covariant

All transporters were assumed to have the same distribution as human BSEP reported in (27); similar expression ranges are also reported in (28); all uptake Vmax values are covariant

All transporters were assumed to have the same distribution as human BSEP reported in (27); similar expression ranges are also reported in (28); all basolateral Vmax values are covariant

All transporters were assumed to have the same distribution as human BSEP reported in (27); similar expression ranges are also reported in (28); all canalicular Vmax values are covariant

All transporters were assumed to have the same distribution as human BSEP reported in (27); similar expression ranges are also reported in (28); all uptake Vmax values are covariant

All transporters were assumed to have the same distribution as human BSEP reported in (27); similar expression ranges are also reported in (28); all basolateral Vmax values are covariant

All transporters were assumed to have the same distribution as human BSEP reported in (27); similar expression ranges are also reported in (28); all canalicular Vmax values are covariant

All transporters were assumed to have the same distribution as human BSEP reported in (27); similar expression ranges are also reported in (28); all uptake Vmax values are covariant

All transporters were assumed to have the same distribution as human BSEP reported in (27); similar expression ranges are also reported in (28); all basolateral Vmax values are covariant

All transporters were assumed to have the same distribution as human BSEP reported in (27); similar expression ranges are also reported in (28); all canalicular Vmax values are covariant 
Table I (continued)

Data used to define parameter distributions (if applicable)

\begin{tabular}{|c|c|c|}
\hline Parameter symbol in DILIsym ${ }^{\circledR}$ & Parameter name in DILlsym ${ }^{\circledR}$ & Data source for distribution \\
\hline CDCA_uptake_Vmax & CDCA uptake $V \max$ & $\begin{array}{l}\text { All transporters were assumed to have the same distribution as hu- } \\
\text { man BSEP reported in (27); similar expression ranges are also } \\
\text { reported in (28); all uptake Vmax values are covariant }\end{array}$ \\
\hline CDCA_baso_Vmax & CDCA basolateral transport $V_{\max }$ & $\begin{array}{l}\text { All transporters were assumed to have the same distribution as hu- } \\
\text { man BSEP reported in (27); similar expression ranges are also } \\
\text { reported in (28); all basolateral Vmax values are covariant }\end{array}$ \\
\hline CDCA_canal_Vmax & CDCA canalicular transport Vmax & $\begin{array}{l}\text { All transporters were assumed to have the same distribution as hu- } \\
\text { man BSEP reported in (27); similar expression ranges are also } \\
\text { reported in (28); all canalicular Vmax values are covariant }\end{array}$ \\
\hline CDCAamide_uptake_Vmax & CDCA-amide uptake Vmax & $\begin{array}{l}\text { All transporters were assumed to have the same distribution as hu- } \\
\text { man BSEP reported in (27); similar expression ranges are also } \\
\text { reported in (28); all uptake Vmax values are covariant }\end{array}$ \\
\hline CDCAamide_baso_Vmax & CDCA-amide basolateral transport $V \max$ & $\begin{array}{l}\text { All transporters were assumed to have the same distribution as hu- } \\
\text { man BSEP reported in (27); similar expression ranges are also } \\
\text { reported in (28); all basolateral Vmax values are covariant }\end{array}$ \\
\hline CDCAamide_canal_Vmax & CDCA-amide canalicular transport $V \max$ & $\begin{array}{l}\text { All transporters were assumed to have the same distribution as hu- } \\
\text { man BSEP reported in (27); similar expression ranges are also } \\
\text { reported in (28); all canalicular Vmax values are covariant }\end{array}$ \\
\hline CDCA_amidation_Vmax & CDCA amidation $V \max$ & Given same range as transporters due to lack of quantitative data \\
\hline LCA_synthesis_Vmax & LCA synthesis Vmax & $\begin{array}{l}\text { Assumed parameter range of } \pm 2 \text { orders of magnitude with } \pm 50 \% \\
\text { standard deviation and validated with outcome data }\end{array}$ \\
\hline LCAamide_sulfation_Vmax & LCA-amide sulfation $V \max$ & Given same range as transporters due to lack of quantitative data \\
\hline canal_reg_scale & Canalicular transporter regulation exponent & $\begin{array}{l}\text { Assumed parameter range of } 0-8 \text { with } \pm 50 \% \text { standard deviation and } \\
\text { validated with outcome data }\end{array}$ \\
\hline uptake_reg_scale & Uptake transporter regulation exponent & $\begin{array}{l}\text { Assumed parameter range of } 0-8 \text { with } \pm 50 \% \text { standard deviation and } \\
\text { validated with outcome data }\end{array}$ \\
\hline
\end{tabular}

For each of the five macrolide antibiotics, the following simulation types were run:

- SimPops simulations: These simulations were conducted using the Human_ROS_apop_mito_BA_v4A_1 SimPops $(n=285)$ included in DILIsym v5A. This SimPops represents variability in parameters related to bile acid homeostasis, mitochondrial function, oxidative stress, apoptosis, and regeneration. A list of parameters varied in the v4A_1 SimPops, as well as the sources used in the construction of the SimPops, are shown in Table I.

- Mechanistic Investigation simulations: These simulations were conducted on a subset of simulated individuals found to be most susceptible to the toxicity from each antibiotic. Then one of the three mechanisms is inactivated for each of the Mechanistic Investigation simulations while the other mechanisms remain active; if inactivating the mechanism leads to a decrease in the number of simulated individuals in which ALT elevations occur, the mechanism is determined to be contributing to the toxicity; the relative magnitude of the decrease in ALT elevation frequency represents the relative contribution of each mechanism to the overall simulated toxicity (16).

\section{RESULTS}

\section{In Vitro Mitochondrial Toxicity Assay Results}

In the mitochondrial respiration assay, solithromycin decreased basal oxygen consumption rate (OCR) in a concentration-dependent manner after 1 and $24 \mathrm{~h}$ incubation, whereas erythromycin did not inhibit cellular respiration at both time points. Telithromycin, clarithromycin, and azithromycin decreased basal OCR following $24 \mathrm{~h}$ incubation, but not after $1 \mathrm{~h}$ incubation (Fig. 2; $1 \mathrm{~h}$ data not shown). These data suggest that all the macrolides tested except for erythromycin are mitochondrial electron transport chain (ETC) inhibitors. Median ratios of intracellular concentration: media concentration measured by LC/MS/MS analysis were 263.4, 24.6, 4.3, 15.2, and 10.5 for solithromycin, 


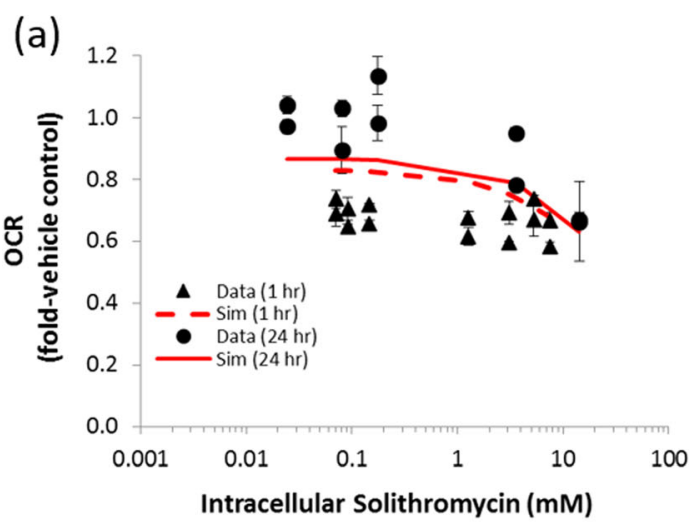

(c)

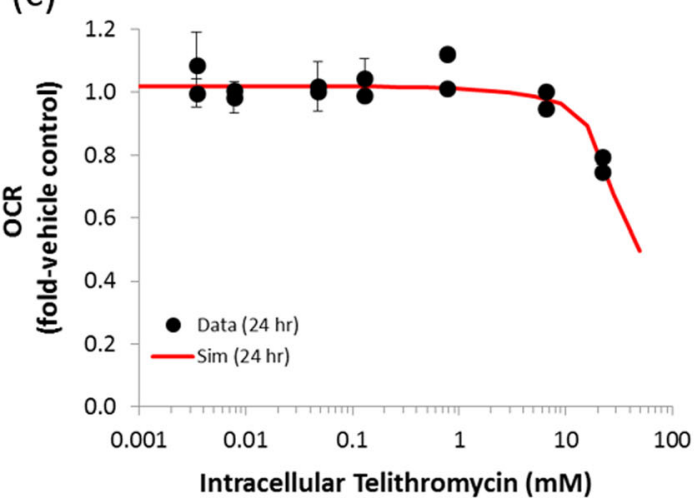

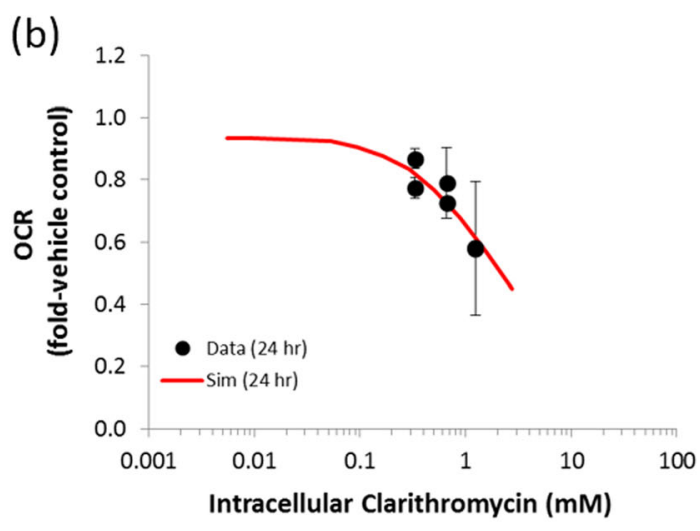

(d)

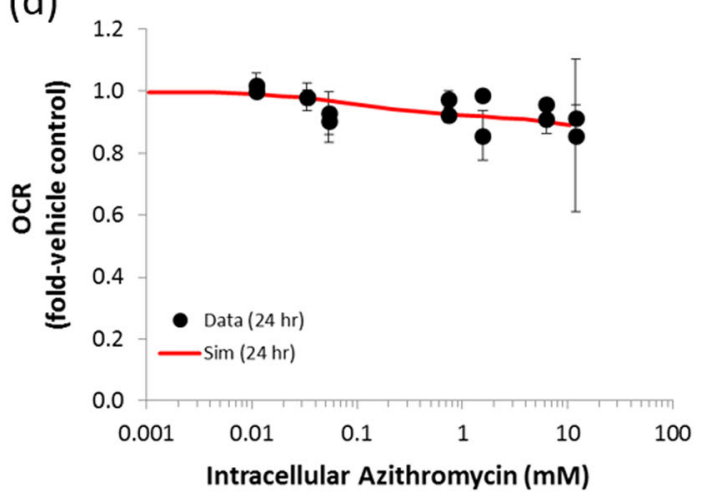

Fig. 2 Comparison of simulation results in in vitro assay data to identify DILIsym parameter values that reproduce the concentration-dependent relationship between macrolides and mitochondrial toxicity. (a) solithromycin I and 24 h, (b) clarithromycin 24 h, (c) telithromycin 24 h, and (d) azithromycin 24 h. Symbols represent the measured oxygen consumption rate (OCR) in each independent experiment, and lines represent the simulated OCR.

erythromycin, telithromycin, clarithromycin, and azithromycin, respectively. DILIsym parameters for mitochondrial ETC inhibition for each compound were optimized to recapitulate intracellular concentrations vs. basal OCR data by simulating in vitro-like conditions within both MITOsym and DILIsym (Fig. 2). Reproduction of the OCR data defined solithromycin, clarithromycin, and azithromycin as ETC inhibitors with both a saturable ETC inhibition at low concentrations and complete ETC inhibition at higher concentrations. DILIsym parameters for complete inhibition (ETC inhibition 1) and saturable inhibition (ETC inhibition 3) were estimated simultaneously for these three compounds using DILIsym, whereas telithromycin's effects on OCR were recapitulated with only the complete inhibition model using MITOsym (Table II).

\section{In Vitro Oxidative Stress Assay Results}

All five macrolides increased RNS/ROS in a concentrationdependent manner after $24 \mathrm{~h}$ incubation, but not following $1 \mathrm{~h}$ incubation (Fig. 3 ; $1 \mathrm{~h}$ data not shown). These data suggest that the tested macrolides can elicit oxidative stress with varying potencies. Median ratios of intracellular concentration: media concentration measured by LC/MS/MS analysis were
78.5, 22.2, 4.34, 3.1, and 297 for solithromycin, erythromycin, telithromycin, clarithromycin, and azithromycin, respectively. DILIsym parameters for production of RNS/ROS were optimized to recapitulate intracellular concentrations vs. cellular RNS/ROS data by simulating in vitro-like conditions within DILIsym (Fig. 3, Table I).

\section{In Vitro Bile Acid Transporter Inhibition Assay Results}

All five macrolides inhibited multiple bile acid transporters with varying potencies. Inhibition constants are presented in Table II.

\section{Simulation Results}

The results for the v4A_1 SimPops simulations for each compound are shown in Table III, compared to the observed clinical frequency of ALT elevations. DILIsym accurately represented the observed frequency of ALT elevations for three of the five macrolide antibiotics. Solithromycin, erythromycin, and clarithromycin were all correctly predicted to cause lowfrequency ALT elevations in the v4A_1 SimPops. The hepatocyte loss in these simulations was not sufficient to cause plasma bilirubin to increase above $2 \mathrm{x}$ the upper limit of normal; 


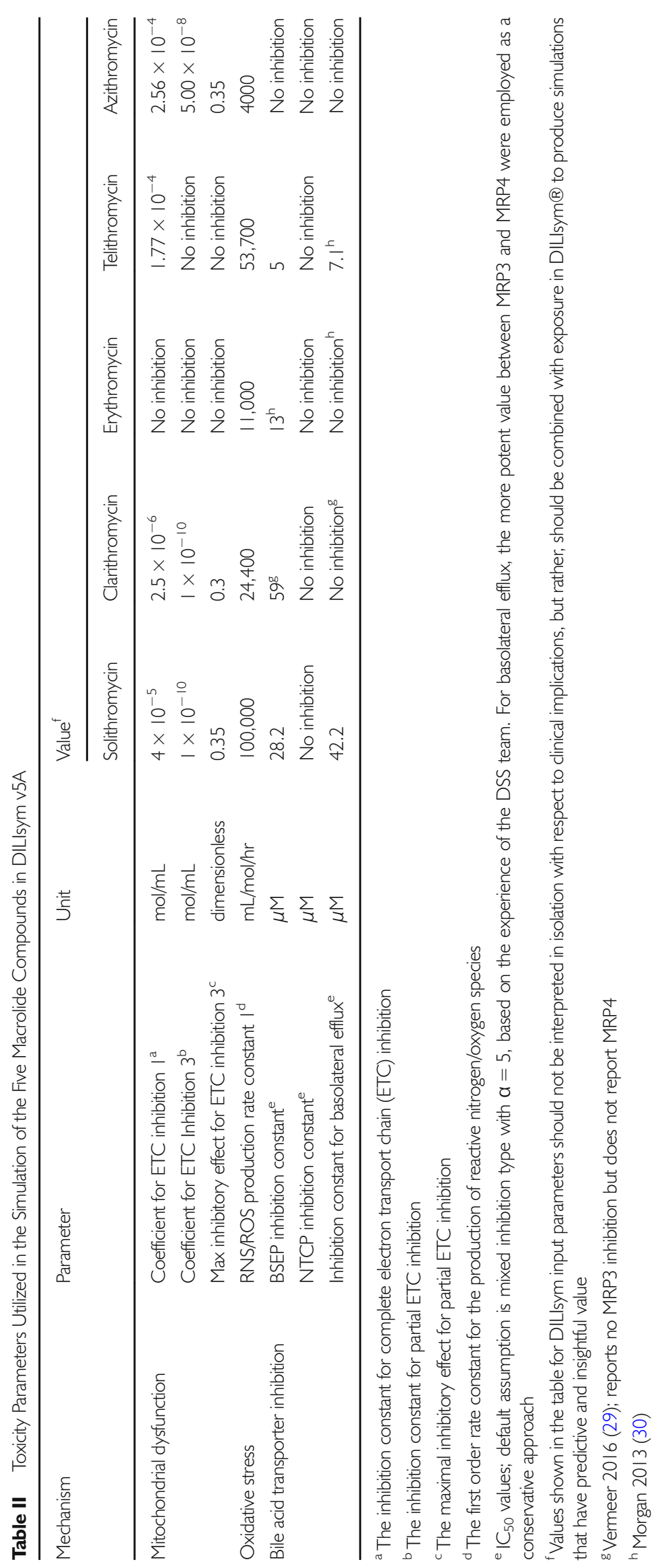



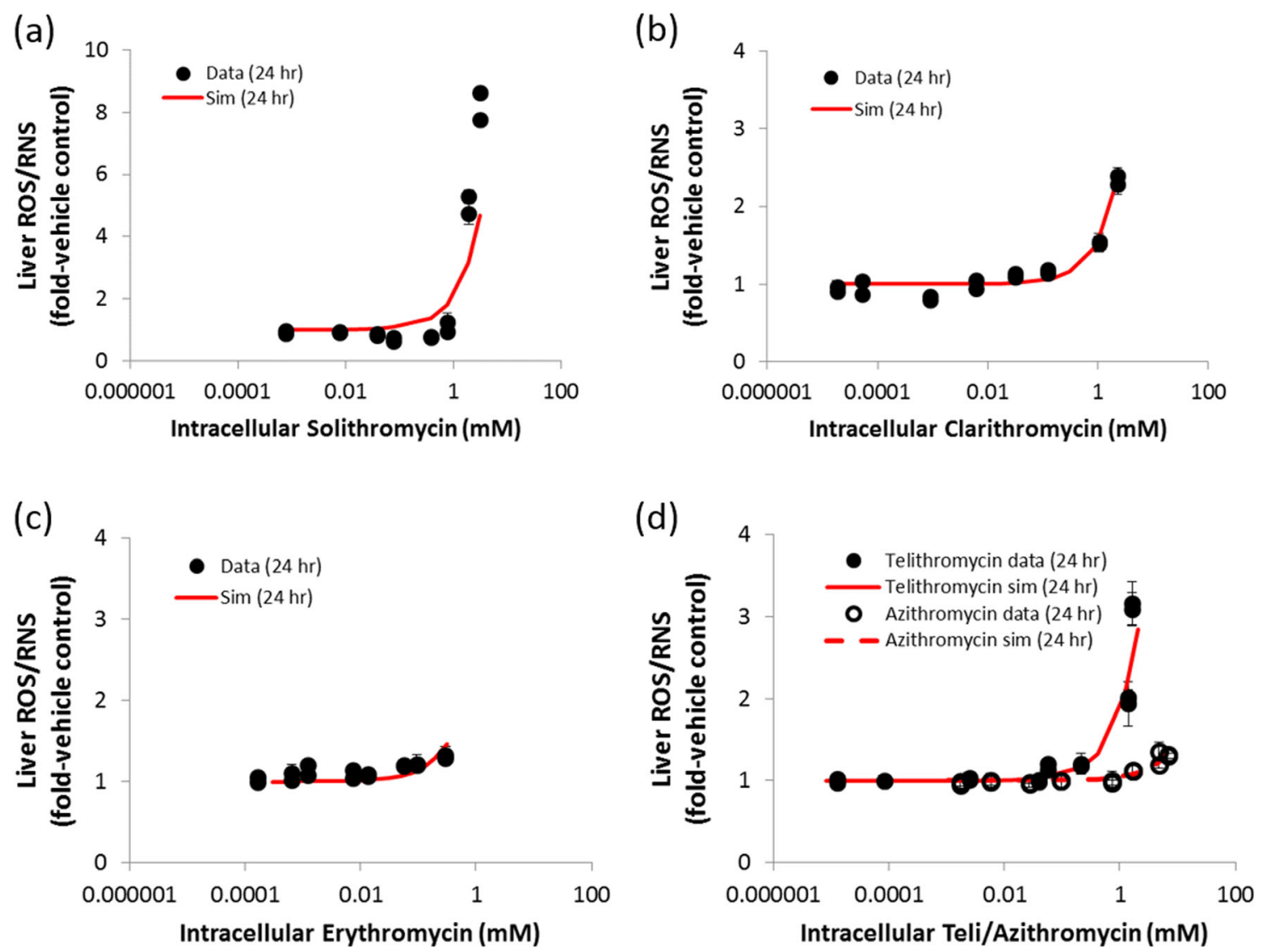

Fig. 3 Comparison of simulation results and in vitro assay data to identify DILIsym parameter values that reproduce the concentration-dependent relationship between macrolides and oxidative stress (a) solithromycin 24 h, (b) clarithromycin 24 h, (c) erythromycin 24 h, and (d) telithromycin and azithromycin 24 h. Symbols represent the measured hepatic reactive oxygen/nitrogen species (ROS/RNS) in each independent experiment, and lines represent the simulated hepatic ROS/RNS.

Table III Results in the v4A_ SimPops for Each of the Five Macrolides in DILIsym v5A Compared to Reported Clinical data. Observed Data are from the Literature $(3,10,31)$

\begin{tabular}{|c|c|c|c|}
\hline \multirow[t]{2}{*}{ Compound } & \multirow[t]{2}{*}{ Protocol } & \multicolumn{2}{|c|}{ Peak ALT > 3X ULN } \\
\hline & & Observed & Simulated ${ }^{* *}$ \\
\hline \multirow[t]{4}{*}{ Solithromycin } & Oral (CEOI-300) & $5.4 \%^{\mathrm{a}}$ & $3.9 \%$ \\
\hline & & $(22 / 4 \mid I)$ & $(1 \mid / 285)$ \\
\hline & IV-to-Oral (CEOI-30I) & $9.1 \%^{b}$ & $6.0 \%$ \\
\hline & & $(38 / 4 \mid 7)$ & $(17 / 285)$ \\
\hline \multirow[t]{2}{*}{ Clarithromycin } & 500 mg BID 7 days & $\mid-2 \%$ & $2.8 \%$ \\
\hline & & & $(8 / 285)$ \\
\hline \multirow[t]{2}{*}{ Erythromycin } & $500 \mathrm{mg}$ & $\mid-2 \%$ & $2.8 \%$ \\
\hline & QID I0 days & & $(8 / 285)$ \\
\hline Telithromycin & 800 mg QD 10 days & $\sim 0.5 \%$ & $0 \%$ \\
\hline Azithromycin & $\begin{array}{l}500 \text { mg QD day I } \\
250 \text { mg QD days 2-5 }\end{array}$ & $1.2 \%$ & $0 \%$ \\
\hline
\end{tabular}

Upper limit of normal (ULN) in DILIsym is $40 \mathrm{U} / \mathrm{L}$

a (9); $2.8 \%$ among patients with normal baseline ALT

b (8); 6.6\% among patients with normal baseline ALT thus, no Hy's Law cases were predicted for these drugs, consistent with clinical data. However, telithromycin and azithromycin were not predicted to cause ALT elevations $>3 \mathrm{X}$ ULN even though some clinical hepatotoxicity has been observed for both of these compounds.

Results for the Mechanistic Investigation Simulations are shown in Table IV. For solithromycin and clarithromycin, the largest decline in simulated ALT elevations was observed when the ETC inhibition effect was omitted, suggesting that this mechanism was the most important in explaining the observed ALT elevations for these two compounds. By contrast, for erythromycin, the elimination of bile acid transporter inhibition effects led to the largest decline in simulated ALT elevation frequency, suggesting that this mechanism best explains the observed erythromycin ALT elevations. For telithromycin and azithromycin, mechanistic investigation simulations were not run since no ALT elevations greater than 3-fold above the ULN occurred in the $\mathrm{v} 4 \mathrm{~A} 1$ SimPops simulations. The likely mechanisms for the five macrolide antibiotics, as predicted by the in vitro assays and the Mechanistic Investigation simulations, are shown in Table V. 
Table IV Mechanistic Investigation Simulations for Each of the Three Macrolides for Which ALT Elevations were Predicted by DILIsym

\begin{tabular}{|c|c|c|c|c|}
\hline \multirow[t]{3}{*}{ Compound } & \multirow[t]{3}{*}{ Mechanism(s) On } & \multirow[t]{3}{*}{ Mechanism Off } & \multicolumn{2}{|c|}{ Simulated } \\
\hline & & & \multicolumn{2}{|c|}{$A L T>3 X \cup L N^{a}$} \\
\hline & & & Oral & IV-to-Oral \\
\hline \multirow[t]{4}{*}{ Solithromycin } & $\begin{array}{l}\text { All } \\
\text { (ETCi, ROS, BAi) }\end{array}$ & - & $11 / 285$ & 17/285 \\
\hline & ETCi, ROS & BAi & $6 / 285$ & $8 / 285$ \\
\hline & BAi, ROS & ETCi & $0 / 285$ & $0 / 285$ \\
\hline & BAi, ETCi & ROS & $11 / 285$ & 17/285 \\
\hline \multirow[t]{3}{*}{ Erythromycin } & $\begin{array}{l}\text { All } \\
\text { (ROS, BAi) }\end{array}$ & - & $8 / 285$ & $\mathrm{~N} / \mathrm{A}$ \\
\hline & ROS & BAi & $1 / 285$ & N/A \\
\hline & BAi & ROS & $7 / 285$ & N/A \\
\hline \multirow[t]{4}{*}{ Clarithromycin } & $\begin{array}{l}\text { All } \\
\text { (ETCi, ROS, BAi) }\end{array}$ & - & $8 / 285$ & $\mathrm{~N} / \mathrm{A}$ \\
\hline & ETCi, ROS & BAi & $3 / 285$ & N/A \\
\hline & BAi, ROS & ETCi & $0 / 285$ & N/A \\
\hline & BAi, ETCi & ROS & $8 / 285$ & $\mathrm{~N} / \mathrm{A}$ \\
\hline
\end{tabular}

For erythromycin, ETC inhibition was not used as a mechanism; this is why there is no simulation with ETCi off

a The upper limit of normal (ULN) of ALT in DILIsym is $40 \mathrm{U} / \mathrm{L}$. Every individual in DILIsym begins the simulation at $30 \mathrm{U} / \mathrm{L}$ plasma ALT

\section{DISCUSSION}

Macrolide antibiotics have been associated with varying levels of liver injury, but the underlying mechanisms have not been elucidated. In the current study, QST modeling was employed to integrate in vitro mechanistic toxicity data, in vivo drug exposure, and underlying biochemistry. Using this approach, DILIsym correctly predicted the frequency of ALT elevations for three of the five macrolides: clarithromycin, solithromycin, and erythromycin. Interestingly, for the three macrolides, different mechanisms were implicated in the observed toxicity; simulations suggest that solithromycin and clarithromycin toxicity is caused mainly by mitochondrial ETC inhibition while erythromycin toxicity is caused predominantly by bile acid accumulation. Furthermore, the fact that telithromycin and azithromycin toxicity was not predicted by DILIsym suggests the presence of a different mechanism that is not represented in DILIsym, though bile acid accumulation did lead to some predictions of sub-clinical ALT elevations for telithromycin (data not shown). This is an interesting result because these five molecules are all in the same class of drug and all somewhat structurally similar; however, they are clearly mechanistically distinct from one another with regard to their hepatic effects. The simulation results therefore demonstrate that one should not draw conclusions about the mechanisms of toxicity - or about the frequency thereof - for a molecule based on the fact that it is part of the same class of drug as another molecule that causes toxicity via a known mechanism.

For telithromycin, in particular, it was not surprising that DILIsym failed to predict ALT elevations. ALT elevations were rather rare in the clinic with telithromycin, and indeed ALT elevations were less frequent during treatment with telithromycin than with the other macrolides (31). The severe toxicity observed with telithromycin was a very rare event (i.e., 1 in 20,000) that may not be able to be predicted in a 285individual simulated population. There are potentially effects caused by metabolites that could have been missed in this analysis; furthermore, if the PBPK simulation underestimated liver partitioning this could also explain an underprediction. As previously mentioned, it is also possible that the cellular stress that occurs with telithromycin may be caused by a mechanism that is not included in DILIsym currently. This is almost certainly the case with azithromycin; while widely considered to be the safest of the macrolide antibiotics $(4,32,33)$, azithromycin has been reported to cause ALT elevations in $1-2 \%$ of the population. However, these elevations often occur after the cessation of dosing $(34,35)$, a phenomenon that cannot be explained by either bile acid accumulation, interference with mitochondrial respiration, or generation of oxidative stress, all of which require drug to be present in order for toxicity to develop. More research should be done to propose a plausible hepatotoxicity mechanism that causes a latent effect that manifests after the cessation of treatment before such a model can be assessed with DILIsym. In the case of telithromycin, inhibition of the "inflammatory reflex" has been proposed as a plausible mechanism for its toxicity based upon its inhibition of nicotinic acetylcholine receptors by the pyridine moiety contained in the side chain of telithromycin $(36,37)$. More recently, the steps following the

Table V Most Likely Mechanism of Toxicity Suggested by the Simulation Results for Each Macrolide Antibiotic

\begin{tabular}{|c|c|c|c|c|c|}
\hline DILI mechanism & Solithromycin & Clarithromycin & Erythromycin & Telithromycin & Azithromycin \\
\hline Mitochondrial dysfunction & Predominant & Predominant & None & None & Plausible \\
\hline Oxidative stress & None & None & Minor & None & None \\
\hline Bile acid transporter inhibition & Minor & Minor & Predominant & Plausible & None \\
\hline Mechanism not included in DILlsym & Unlikely & Unlikely & Unlikely & Plausible & Plausible \\
\hline
\end{tabular}

The mechanism suggested by DILIsym as the most likely to contribute to the observed toxicity is rendered in bold 
release of acetylcholine, activation of the $\mathrm{nACh}$ receptors, and hepatocyte regeneration has been described (38). Blocking of $\mathrm{nACh}$ receptor activation by telithromycin would block protective hepatocyte regeneration.

One of the more interesting aspects of this work is how the in vitro assays did not map directly to the mechanisms that were responsible for toxicity. For example, each of the five macrolides demonstrated some response in the oxidative stress assay, but ROS was found to contribute only to erythromycin toxicity by the simulations. The combination of in vitro assay results with measures of exposure has been shown to produce an increased ability to predict liver toxicity beyond that of the assay alone (30); the combination of in vitro assay results, exposure estimates, and known biological variability contained by QST models such as DILIsym can provide considerably greater mechanistic insight than the assays results alone.

In patients treated with solithromycin, increased ALT was normalized with continued dosing or soon after the end of treatment (8). These data suggest that the liver was able to adapt to the mild liver injury instigated by solithromycin. One proposed mechanism for adaptation in liver injury is mitochondrial biogenesis; this is a key mechanism for recovery from mitochondrial stress in muscles resulting from exercise (39) and has been observed in mouse hepatocytes after exposure to the ETC inhibitor rotenone (40) and in rat liver after dosing with the DILI-inducing drug valproate $(41,42)$. Solithromycin and clarithromycin, as drugs whose ALT elevations are largely driven by mitochondrial effects, would be more likely to respond with this adaptive mechanism, which may be less relevant to the other macrolides. DILIsym does not yet include mitogenesis and several other potentially important adaptive processes that may mitigate some of the toxic response to drugs. Future simulation work will incorporate mitochondrial biogenesis into DILIsym and compare the effects of this adaptive mechanism on the simulation results.

One limitation of this work is that the metabolites of the macrolides were not investigated for their potential toxic effects. The results suggest that it is unlikely that metabolites of solithromycin, clarithromycin, and erythromycin are contributing to the observed ALT elevations; the liver responses for these molecules were adequately explained by the effects of the parent compound. However, metabolite effects may be contributing to azithromycin and telithromycin toxicity, since these compounds' toxic responses were not adequately explained by parent effects due to the mechanisms represented in DILIsym. Furthermore, the simulations were conducted in a SimPops intended to represent normal healthy volunteers; the ALT elevations were almost all observed in individuals with some sort of bacterial infection. It is unclear whether infected individuals demonstrate a different level of drug exposure for many of the macrolides, though elderly individuals and individuals with communityacquired bacterial pneumonia have been shown to have a higher plasma concentration of telithromycin than normal healthy volunteers (43). Differences in exposure between healthy volunteers and infected individuals may help explain some of the observed telithromycin and azithromycin toxicity; a better understanding of the differences in exposure between infected and healthy individuals is necessary. Uncertainty in the in vitro-in vivo extrapolation (IVIVE) process for the toxicity parameters is also a plausible reason for the lack of predictivity for telithromycin and azithromycin; if the estimate of intracellular:extracellular concentration ratio derived from the mass spectrometry assay is significantly different from that which occurs in vivo, for example, the toxicity parameter derivation may be affected as a result. However, the magnitude of the assay uncertainty necessary for this effect to be able to explain the missed predictions of telithromycin and azithromycin is quite large, as suggested by dose escalation simulations conducted on both compounds (data not shown). As a result, we view this as a less likely contributor than alternative mechanisms, patient effects, or metabolite effects.

\section{CONCLUSIONS}

In conclusion, DILIsym was used to contextualize novel in vitro experiments and assess the likelihood that five macrolide antibiotics cause serum ALT elevations by oxidative stress, mitochondrial toxicity, and bile acid accumulation. DILIsym found that solithromycin and clarithromycin ALT elevations can be primarily accounted for by mitochondrial ETC inhibition whereas erythromycin ALT elevations can be primarily accounted for by inhibition of bile acid transporters. Bile acid transporter inhibition may also in part account for ALT elevations caused by telithromycin, but the model predictions were poor for both telithromycin and azithromycin. This may reflect effects of metabolites of these drugs or involvement of mechanisms not included in DILIsym. This research demonstrates that despite the fact that these five drugs are all in the same class, they are mechanistically distinct from one another concerning their hepatic adverse effects.

\section{ACKNOWLEDGMENTS AND DISCLOSURES}

Drs. Oldach, MacLauchlin, and Fernandes were previously employees of Cempra, Inc., the manufacturer of solithromycin. Drs. Woodhead, Yang, Siler, and Howell are employees of DILIsym Services, Inc., the manufacturer of the DILIsym software package. Dr. Watkins served as a paid consultant to Cempra, Inc. The authors acknowledge the contributions of the members of the DILI-sim Initiative, under whose auspices DILIsym was developed. Information on Initiative membership and access to the DILIsym software is provided at https://www.simulations-plus.com/software/ dilisym/. 
Open Access This article is distributed under the terms of the Creative Commons Attribution 4.0 International License (http://creativecommons.org/licenses/by/4.0/), which permits unrestricted use, distribution, and reproduction in any medium, provided you give appropriate credit to the original author(s) and the source, provide a link to the Creative Commons license, and indicate if changes were made.

Publisher's Note Springer Nature remains neutral with regard to jurisdictional claims in published maps and institutional affiliations.

\section{REFERENCES}

1. Albert RK, Schuller JL, COPD Clinical Research Network. Macrolide antibiotics and the risk of cardiac arrhythmias. Am J Respir Crit Care Med. 2014;189(10):1173-80.

2. Cervin A, Wallwork B. Efficacy and safety of long-term antibiotics (macrolides) for the treatment of chronic rhinosinusitis. Curr Allergy Asthma Rep. 2014;14(3):416.

3. Macrolide Antibiotics [Internet]. [cited 2018 Jan 4]. Available from: https://livertox.nih.gov/MacrolideAntibiotics.htm. Accessed 27 Mar 2018.

4. Andrade RJ, Tulkens PM. Hepatic safety of antibiotics used in primary care. J Antimicrob Chemother. 201 1;66(7):1431-46.

5. Brinker AD, Wassel RT, Lyndly J, Serrano J, Avigan M, Lee WM, et al. Telithromycin-associated hepatotoxicity: clinical spectrum and causality assessment of 42 cases. Hepatol. 2009;49(1):250-7.

6. Robles M, Toscano E, Cotta J, Lucena MI, Andrade RJ. Antibiotic-induced liver toxicity: mechanisms, clinical features and causality assessment. Curr Drug Saf. 2010;5(3):212-22.

7. Telithromycin [Internet]. DrugBank; [cited 2016 Nov 1]. Available from: https://www.drugbank.ca/drugs/DB00976. Accessed 27 Mar 2018.

8. File TM, Rewerska B, Vucinic-Mihailovic V, Gonong JRV, Das AF, Keedy K, et al. SOLITAIRE-IV: a randomized, double-blind, multicenter study comparing the efficacy and safety of intravenousto-oral solithromycin to intravenous-to-oral moxifloxacin for treatment of community-acquired bacterial pneumonia. Clin Infect Dis. 2016;63(8):1007-16.

9. Barrera CM, Mykietiuk A, Metev H, Nitu MF, Karimjee N, Doreski PA, et al. Efficacy and safety of oral solithromycin versus oral moxifloxacin for treatment of community-acquired bacterial pneumonia: a global, double-blind, multicentre, randomised, active-controlled, non-inferiority trial (SOLITAIRE-ORAL). Lancet Infect Dis. 2016;16(4):421-30.

10. Solithromycin Oral Capsule and Injection: Meeting of the Antimicrobial Drugs Advisory Committee [Internet]. 2016 [cited 2018 Jan 4]. Available from: https://www.fda.gov/downloads/ AdvisoryCommittees/CommitteesMeetingMaterials/Drugs/AntiInfectiveDrugsAdvisoryCommittee/UGM527690.pdf. Accessed 18 Apr 2018.

11. FDA Rejects NDAs for Cempra Bacterial Pneumonia Candidate Solithromycin [Internet]. GEN. [cited 2018 Feb 19]. Available from: https://www.genengnews.com/gen-news-highlights/fdarejects-ndas-for-cempra-bacterial-pneumonia-candidatesolithromycin/81253623. Accessed 18 Apr 2018.

12. Bloomingdale P, Housand C, Apgar JF, Millard BL, Mager DE, Burke JM, et al. Quantitative systems toxicology. Curr Opin Toxicol. 2017;4:79-87.
13. Howell BA, Yang Y, Kumar R, Woodhead JL, Harrill AH, Clewell $\mathrm{HJ}$ 3rd, et al. In vitro to in vivo extrapolation and species response comparisons for drug-induced liver injury (DILI) using DILIsym ${ }^{\mathrm{TM}}$ : a mechanistic, mathematical model of DILI. J Pharmacokinet Pharmacodyn. 2012;39(5):527-41.

14. Shoda LKM, Woodhead JL, Siler SQ, Watkins PB, Howell BA. Linking physiology to toxicity using DILIsym( $\left({ }^{\circledR}\right)$, a mechanistic mathematical model of drug-induced liver injury. Biopharm Drug Dispos. 2014;35(1):33-49.

15. Woodhead JL, Watkins PB, Howell BA, Siler SQ Shoda LKM. The role of quantitative systems pharmacology modeling in the prediction and explanation of idiosyncratic drug-induced liver injury. Drug Metab Pharmacokinet. 2017;32(1):40-5.

16. Woodhead JL, Brock WJ, Roth SE, Shoaf SE, Brouwer KLR, Church R, et al. Application of a mechanistic model to evaluate putative mechanisms of tolvaptan drug-induced liver injury and identify patient susceptibility factors. Toxicol Sci. 2017;155(1):6174 .

17. Woodhead JL, Yang K, Siler SQ, Watkins PB, Brouwer KLR, Barton HA, et al. Exploring BSEP inhibition-mediated toxicity with a mechanistic model of drug-induced liver injury. Front Pharmacol. 2014;5:240.

18. Yang Y, Nadanaciva S, Will Y, Woodhead JL, Howell BA, Watkins $\mathrm{PB}$, et al. MITOsym ${ }^{\circledR}$ : a mechanistic, Mathematical Model of Hepatocellular Respiration and Bioenergetics. Pharm Res. 2015;32:1975-92. https://doi.org/10.1007/s1 1095-014-1591-0.

19. Longo DM, Yang Y, Watkins PB, Howell BA, Siler SQ. Elucidating differences in the hepatotoxic potential of tolcapone and entacapone with DILIsym( $(\mathbb{B})$, a mechanistic model of druginduced liver injury. CPT Pharmacometrics Syst Pharmacol. 2016;5(1):31-9.

20. Woodhead JL, Howell BA, Yang Y, Harrill AH, Clewell HJ 3rd, Andersen ME, et al. An analysis of N-acetylcysteine treatment for acetaminophen overdose using a systems model of drug-induced liver injury. J Pharmacol Exp Ther. 2012;342(2):529-40.

21. Allen JW, Shanker G, Aschner M. Methylmercury inhibits the in vitro uptake of the glutathione precursor, cystine, in astrocytes, but not in neurons. Brain Res. 2001;894(1):131-40.

22. Lee K-T, Tsai S-M, Wang S-N, Lin S-K, Wu S-H, Chuang S-C, et al. Glutathione status in the blood and tissues of patients with virus-originated hepatocellular carcinoma. Clin Biochem. 2007;40(15):1157-62.

23. Nagasaka H, Takayanagi M, Tsukahara H. Children's toxicology from bench to bed-liver injury (3): oxidative stress and anti-oxidant systems in liver of patients with Wilson disease. J Toxicol Sci. 2009;34(Suppl 2):SP229-36.

24. Shon Y-H, Nam K-S. Protective effect of moutan cortex extract on acetaminophen-induced cytotoxicity in human Chang liver cells. Am J Health Syst Pharm. 2002;25(11):1427-31.

25. Pérez-Carreras M, Del Hoyo P, Martín MA, Rubio JC, Martín A, Castellano G, et al. Defective hepatic mitochondrial respiratory chain in patients with nonalcoholic steatohepatitis. Hepatol Baltim Md. 2003;38(4):999-1007.

26. Bantel H, Ruck P, Gregor M, Schulze-Osthoff K. Detection of elevated caspase activation and early apoptosis in liver diseases. Eur J Cell Biol. 2001;80(3):230-9.

27. Meier Y, Pauli-Magnus C, Zanger UM, Klein K, Schaeffeler E, Nussler AK, et al. Interindividual variability of canalicular ATPbinding-cassette $(\mathrm{ABC})$-transporter expression in human liver. Hepatol Baltim Md. 2006;44(1):62-74.

28. Bernhardt GA, Zollner G, Cerwenka H, Kornprat P, Fickert P, Bacher $\mathrm{H}$, et al. Hepatobiliary transporter expression and postoperative jaundice in patients undergoing partial hepatectomy. Liver Int Off J Int Assoc Study Liver. 2012;32(1):119-27.

29. Vermeer LMM, Isringhausen CD, Ogilvie BW, Buckley DB. Evaluation of ketoconazole and its alternative clinical CYP3A4/5 
inhibitors as inhibitors of drug transporters: the in vitro effects of ketoconazole, ritonavir, clarithromycin, and itraconazole on 13 clinically-relevant drug transporters. Toxicol Sci. 2016;44(3):4539.

30. Morgan RE, van Staden CJ, Chen Y, Kalyanaraman N, Kalanzi J, Dunn RT, et al. A multifactorial approach to hepatobiliary transporter assessment enables improved therapeutic compound development. J Appl Physiol. 2013;136(1):216-41.

31. Kaye JA, Castellsague J, Bui CL, Calingaert B, McQuay LJ, RieraGuardia N, et al. Risk of acute liver injury associated with the use of moxifloxacin and other oral antimicrobials: a retrospective, population-based cohort study. Pharmacotherapy. 2014;34(4): 336-49.

32. Mertens JC, van Barneveld PW, Asin HR, Ligtvoet E, Visser MR, Branger $\mathrm{T}$, et al. Double-blind randomized study comparing the efficacies and safeties of a short (3-day) course of azithromycin and a 5-day course of amoxicillin in patients with acute exacerbations of chronic bronchitis. Antimicrob Agents Chemother. 1992;36(7):1456-9.

33. Gašparić M, Penezić A, Kolumbić-Lakoš A, Kovačić D, Kukuruzović MM, Baršić B. Safety and effectiveness of azithromycin in the treatment of lower respiratory infections: an international, multicenter, non-comparative study. Acta Clin Croat. 2015;54(2): 149-58.

34. Lockwood AM, Cole S, Rabinovich M. Azithromycin-induced liver injury. Am J Health-Syst Pharm AJHP Off J Am Soc Health-Syst Pharm. 2010;67(10):810-4.

35. Martinez MA, Vuppalanchi R, Fontana RJ, Stolz A, Kleiner DE, Hayashi PH, et al. Clinical and histologic features of azithromycininduced liver injury. Clin Gastroenterol Hepatol Off Clin Pract J Am Gastroenterol Assoc. 2015;13(2):369-376.e3.
36. Bertrand D, Bertrand S, Neveu E, Fernandes P. Molecular characterization of off-target activities of telithromycin: a potential role for nicotinic acetylcholine receptors. Antimicrob Agents Chemother. 2010;54(12):5399-402.

37. Tracey KJ. The inflammatory reflex. Nature. 2002;420(6917):8539.

38. Izumi T, Imai J, Yamamoto J, Kawana Y, Endo A, Sugawara H, et al. Vagus-macrophage-hepatocyte link promotes post-injury liver regeneration and whole-body survival through hepatic FoxMl activation. Nat Commun [Internet]. 2018 Dec 13 [cited 2018 Dec 27];9. Available from: https://www.ncbi.nlm.nih.gov/pmc/ articles/PMC6294142/. Accessed 27 Dec 2018.

39. Hood DA. Invited Review: contractile activity-induced mitochondrial biogenesis in skeletal muscle. Hepatol. 1985. 2001;90(3): 1137-57.

40. Than TA, Lou H, Ji C, Win S, Kaplowitz N. Role of cAMPresponsive element-binding protein (CREB)-regulated transcription coactivator 3 (CRTC3) in the initiation of mitochondrial biogenesis and stress response in liver cells. J Biol Chem. 201 1;286(25): 22047-54.

41. Hayasaka K, Takahashi I, Kobayashi Y, linuma K, Narisawa K, Tada K. Effects of valproate on biogenesis and function of liver mitochondria. Hepatol. 1986;36(3):351-6.

42. Sitarz KS, Elliott HR, Karaman BS, Relton C, Chinnery PF, Horvath R. Valproic acid triggers increased mitochondrial biogenesis in POLG-deficient fibroblasts. Liver Int. 2014;112(1):57-63.

43. Shi J, Montay G, Bhargava VO. Clinical pharmacokinetics of telithromycin, the first ketolide antibacterial. Clin Pharmacokinet. 2005;44(9):915-34. 